\title{
Interleukin-17F expression is elevated in hepatitis $C$ patients with fibrosis and hepatocellular carcinoma
}

\author{
Ming-Sian $\mathrm{Wu}^{1 \dagger}{ }^{1 \dagger}$ Chun-Hsiang Wang ${ }^{2 \dagger}$, Fan-Chen Tseng ${ }^{1}$, Hsuan-Ju Yang ${ }^{2}$, Yin-Chiu Lo ${ }^{1}$, Yi-Ping Kuo ${ }^{1}$, \\ De-Jiun Tsai ${ }^{1}$, Wan-Ting Tsai ${ }^{1}$ and Guann-Yi Yu ${ }^{1,3^{*}}$
}

\begin{abstract}
Background: The role of interleukin (IL) 17A in chronic liver diseases had been extensively studied, but the function of IL-17F, which shares a high degree of homology with IL-17A, in the progression of chronic hepatic diseases is poorly understood. The aim of the study was to evaluate the association between IL-17F and liver diseases including, fibrosis and hepatocellular carcinoma (HCC).

Methods: Hepatic tumor samples from both hepatitis C virus $(\mathrm{HCV}$ ) positive and negative patients (without HBV and $\mathrm{HCV}, \mathrm{NBNC}$ ) were examined with quantitative PCR and immunohistochemistry staining for inflammatory cytokine genes expression. In addition, $250 \mathrm{HCV}$ patients naïve for interferon treatment were also subjected to enzyme-linked immunosorbent Assay (ELISA) for their serum cytokine concentrations.
\end{abstract}

Results: Serum IL-17F concentrations were significantly elevated in HCV patients with severe fibrosis stages. In accordance with serum data, IL-17F expression was also found higher in HCV-associated HCC tissues compared with NBNC HCC tissues at both the mRNA and protein levels.

Conclusions: Our data suggest that IL-17F might be used as a valuable biological marker than IL-17A during chronic fibrosis progression and HCC development in HCV patients.

Keywords: I-17F, Hepatitis C virus, Fibrosis, Hepatocellular carcinoma

\section{Background}

Approximately 80 million people worldwide have viremic hepatitis $\mathrm{C}$ virus $(\mathrm{HCV})$ infection, which causes chronic hepatitis, fibrosis, cirrhosis, and hepatocellular carcinoma (HCC) [1]. The full progression of end-stage liver diseases in HCV-infected patients takes about two to three decades, which provides a window for intervention. Liver fibrosis is a protective response to chronic hepatic injury that leads to accumulation of extracellular matrix proteins [2]. Extensive fibrosis may result in cirrhosis and, in severe cases, lead to liver failure requiring liver transplantation. Severe fibrosis and cirrhosis are the outcome of

\footnotetext{
* Correspondence: guannyiy@nhri.org.tw

'Equal contributors

${ }^{1}$ National Institute of Infectious Diseases and Vaccinology, National Health Research Institutes, 35 Keyan Road, Zhunan, Miaoli County 35053, Taiwan

${ }^{3}$ Center of Infectious Disease and Signaling Research, National Cheng-Kung University, Tainan 70101, Taiwan

Full list of author information is available at the end of the article
}

continuous liver injuries, which can be caused by chronic virus infection or long-term alcohol consumption. Liver fibrosis is one of the known risk factors for HCC [3]. Chronic inflammation caused by innate and adaptive immune responses to HCV infection is involved in the progression of $\mathrm{HCV}$-associated diseases, such as liver cirrhosis and HCC $[4,5]$. Identification of factors involved in $\mathrm{HCV}$ pathogenesis or biomarkers associated with liver diseases may provide new intervention and treatment approaches for HCV-related diseases.

$\mathrm{T}$ helper (Th) 17 cells are a subset of $\mathrm{CD}^{+} \mathrm{T}$ cells that mediate a protective role against bacterial and fungal infections $[6,7]$ as well as a pathological role in inflammation-associated diseases, such as autoimmune diseases and cancer [8-10]. Transforming growth factor (TGF)- $\beta$, interleukin (IL)-6, IL-21, and IL-23 are the key cytokines for Th17 cell maturation and production of IL-17 family cytokines, the secretion of which is a 
quintessential defining feature for Th17 cells [11-13]. The IL-17 family has six members, including IL-17A-F. IL-17A and IL-17F have high sequence homology and are expressed as homodimers or as an IL-17A + F heterodimer to induce expression proinflammatory cytokines, chemokines, antimicrobial peptides, and matrix metalloproteinases in IL-17 receptor-bearing cells [14].

Th17 cells play an important role in many liver diseases, such as liver fibrosis, alcoholic liver disease, chronic hepatitis B, and autoimmune liver disease [15-18]. The Th17 cell population is increased in chronic hepatitis $C$ patients and Th17 cell abundance correlates positively with liver injury but inversely with HCV RNA load [19]. Although IL-17A had been extensively studied in chronic liver diseases, the function of IL-17F, which shares a high degree of homology with IL-17A, in the progression of chronic hepatic diseases is poorly understood. IL-17F can be secreted by $\mathrm{CD}^{+} \mathrm{T}$ cells, $\gamma \delta \mathrm{T}$ cells, NKT cells, LTi-like cells, and epithelial cells in addition to being secreted by Th17 cells $\left(\mathrm{CD}^{+}{ }^{+} \mathrm{T}\right.$ cells) $[8,20]$. IL-17F has a weaker receptor binding affinity than IL-17A and therefore induces less expression of proinflammatory cytokines than IL-17A [21]. The aim of this study was to evaluate the association between the expression of IL-17F and HCV-associated diseases by the evaluation of Th17-related cytokine expression in $\mathrm{HCC}$ tissue and serum from $\mathrm{HCV}$ patients.

\section{Methods}

\section{HCC cohort}

RNA samples derived from cancerous and non-cancerous parts of HCC tissue from patients infected with $\mathrm{HCV}$ and controls negative for both HBV and HCV ("non-B, nonC", NBNC) were obtained from the Taiwan Liver Cancer Network (TLCN) [22].

\section{Treatment naïve HCV cohort}

A total of 250 patients who had serologically demonstrated $\mathrm{HCV}$ infection and were treated at Tainan Municipal Hospital (TMH), Tainan, Taiwan from 2003 to 2008 were included in our serum cytokine analysis. Baseline sera were collected from the patients within two months before they underwent $\mathrm{HCV}$ antiviral therapy. Patients infected with HBV or HIV were excluded from the study. The diagnoses of hepatic steatosis, fibrosis, and HCC of the HCV cohort were independently confirmed by two pathologists at TMH. Based on the METAVIR grading system, liver fibrosis status was classified as: no fibrosis (F0), portal fibrosis without septa (F1), with few septa (F2), with numerous septa but without cirrhosis (F3), and with cirrhosis (F4).

\section{Serum cytokine detection}

IL-6, IL-17A, IL-17F, and IL-21 concentrations in serum samples were measured by enzyme-linked immunosorbent assay (ELISA) according to the manufacturer's instructions. Human IL-6, IL-17A, and IL-17F ELISA kits were purchased from R\&D Systems (MN, USA), and the IL21 immunoassay kit was obtained from eBioscience (CA, USA). The kits' analytic sensitivities were $9.375 \mathrm{pg} /$ $\mathrm{mL}$ for IL-6, $15.625 \mathrm{pg} / \mathrm{mL}$ for IL-17A, $312.5 \mathrm{pg} / \mathrm{mL}$ for IL-17F, and $8 \mathrm{pg} / \mathrm{mL}$ for IL-21.

\section{Cytokine mRNA quantification}

Total RNA samples $(1 \mu \mathrm{g})$ extracted from HCC cancerous and non-cancerous tissues were used for cDNA synthesis with SuperScript-III First-Strand Synthesis System (Invitrogen, Carlsbad, CA). cDNAs were then used as templates in the quantitative-polymerase chain reaction (PCR) with gene specific primers and SYBR green dye to determine quantification cycle (Cq) by Applied Biosystems 7900HT Fast Real-Time PCR System. Primer sequences for human cytokine IL-17A and IL-17F and housekeeping gene ubiquitin C (UBC) [23] were listed in Table 1. Relative cytokine mRNA expression level was normalized to UBC reference gene by the $2^{-\Delta \Delta C q}$ method [24]. Cytokine mRNA expression with $\mathrm{Cq}<35$ cycles was counted as positive, and the positive frequency was determined for cancerous and non-cancerous tissue separately for each group.

\section{IL-17F immunohistochemistry}

Paraffin-embedded sections of the HCC cohort were subjected to immunohistochemistry (IHC) by following the protocol described previously with minor modification [25]. In brief, paraffin-embedded sections were deparaffinized and incubated in citrate buffer $(\mathrm{pH} 6)$ with $0.05 \%$ Tween 20 at $95-98{ }^{\circ} \mathrm{C}$ for $20 \mathrm{~min}$. After $3 \%$ $\mathrm{H}_{2} \mathrm{O}_{2}$ and $1 \%$ bovine serum albumin blocking, slides were incubated with the anti-IL-17F antibody (ab168194, Abcam) overnight at $4{ }^{\circ} \mathrm{C}$, followed by incubation with a secondary antibody (EnVision ${ }^{+}$system-HRP-labeled polymer, DakoCytomation) at room temperature for $1 \mathrm{~h}$. The sections were then stained with 3,3'-diaminobenzidine substrate and counterstained with hematoxylin. Samples were considered IL-17F-immunopositive if at least $10 \%$ of randomly selected fields contained positive staining signals.

Table 1 Primer sequences for quantitative RT-PCR

\begin{tabular}{ll}
\hline Gene Name & Oligo sequences \\
\hline UBC forward & 5' CCTGGTGCTCCGTCTTAGAG 3' \\
UBC reverse & 5' TाTCCCAGCAAAGATCAACC 3' \\
IL-17A forward & 5' AATCTCCACCGCAATGAGGA 3' \\
IL-17A reverse & 5' ACGTTCCCATCAGCGTTGA 3' \\
IL-17F forward & 5' GAAGCTTGACATTGGCATCA 3' \\
IL-17F reverse & 5' GATGCAGCCCAAGTCCTAC 3' \\
\hline
\end{tabular}


Table 2 Demographic Summary of TLCN HCC cohort

\begin{tabular}{lll}
\hline Variable & $\operatorname{HCV}(n=40)$ & $\operatorname{NBNC}(n=32)$ \\
\hline Age & $66.3 \pm 8.4(59.3-72.0)$ & $66.0 \pm 14.9(61.0-76.0)$ \\
Gender & Male:32, Female:8 & Male:22, Female:10 \\
Presence of cirrhosis & $45.0 \%$ & $21.9 \%$ \\
Tumor size (cm) & $4.5 \pm 2.3(3.0-5.9)$ & $8.0 \pm 5.1(3.5-11.8)$ \\
Positive frequency ${ }^{\mathrm{a}}$ & $($ Tumor, Non-Tumor) & (Tumor, Non-Tumor) \\
$\quad$ IL-17A & $80.0 \%, 75.0 \%$ & $87.5 \%, 87.5 \%$ \\
$\quad$ IL-17F & $47.5 \%, 15.0 \%$ & $40.6 \%, 18.8 \%$ \\
\hline
\end{tabular}

${ }^{a}$ Specific cytokine gene expression was detected by quantitative RT-PCR

\section{Statistical analyses}

Statistical analyses were performed with SPSS software (IBM, NY, USA). Serum cytokine and mRNA expression were across-analyzed with demographic and clinical groups using the Mann-Whitney $U$ test and Kruskal-Wallis tests. The relative distribution of IL-17A and IL-17F mRNA expression in cancerous and noncancerous tissue was analyzed by the Fisher exact test and Mann-Whitney $U$ test. In all cases, $p<0.05$ was considered statistically significant.

\section{Results}

IL-17F expression was elevated in HCV-associated HCC

Table 2 listed that the characteristics of the $\operatorname{HCV}(N=40)$ and NBNC $(N=32)$ cancer patients from whom paired cancerous and adjacent non-cancerous tissues from TLCN tissue bank were obtained and subjected to quantitative RT-PCR analysis with SYBR dye for IL-17A and IL17F mRNA detection. IL-17A mRNA was present in more than $75 \%$ of RNA samples derived from both cancerous and non-cancerous tissues. In contrast, IL-17F mRNA was expressed only in a portion of HCC samples. As shown in Table 2, the positive frequency was higher in cancerous tissue than in non-cancerous tissue, and notably higher in HCV-infected tissue (47.5\% vs. $15 \%$, $p=0.003$ by Fisher exact test) than in NBNC patients ( $40.6 \%$ vs. $18.8 \%, p=0.1$ by Fisher exact test). Relative IL17A mRNA expression level demonstrated no differences between cancerous and non-tumor counterpart tissues either derived from $\mathrm{HCV}$-infected patients or NBNC patients. The relative IL-17A and F mRNA expression level in those positive cases did not show a significant difference between HCV and NBNC patients.

IHC revealed IL-17F-immunopositive signals in hepatocytes in HCC tissues (Fig. 1). In total, $85 \%$ of the HCC tumor samples from the HCV group and $62 \%$ of the HCC tumor samples from NBNC group were positive for IL$17 F$ staining ( $p=0.0002$ by Fisher exact test). In summary, IL-17F expression was higher in $\mathrm{HCV}$-associated HCC tissues compared with NBNC HCC tissues at both the mRNA and protein levels.

\section{Association of serum IL-17F level with liver fibrosis progression in treatment naïve HCV patient cohort} Due to HCC development is largely confined to patients with liver fibrosis and cirrhosis, a retrospective cohort study was further conducted to determine serum IL-17F expression levels in $\mathrm{HCV}$ patients with chronic hepatic diseases. Diagnosis of $\mathrm{HCV}$ infection was confirmed in all $250 \mathrm{HCV}$ patients by two sequential tests for $\mathrm{HCV}$ antibodies, followed by an HCV RNA assay. The data consisted of 127 men (51\%) and 123 women (49\%) with

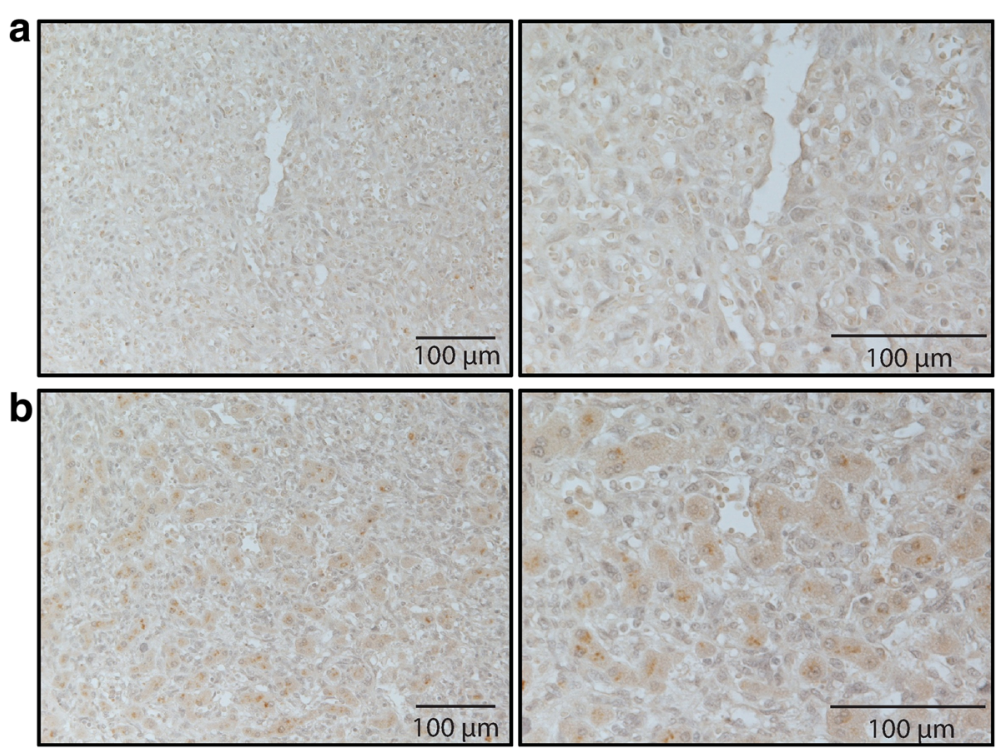

Fig. 1 Representative images of IL-17F immunohistochemistry staining. Representative images for IL-17F IHC labeling in HCV-infected HCC tumor tissue samples without (a) and with (b) IL-17F immunopositivity. Magnification in the left column-200X and in the right column-400X 
Table 3 Variables of HCV infected patients from TMH

\begin{tabular}{|c|c|c|c|c|}
\hline \multirow{2}{*}{\multicolumn{2}{|c|}{$\begin{array}{l}\text { Variable } \\
\text { Age }\end{array}$}} & \multirow{2}{*}{$\frac{N}{250}$} & \multirow{2}{*}{$\begin{array}{l}\text { Mean (IQR) } \\
56.6 \pm 10.5 \\
(51.5-64.8)\end{array}$} & \multirow[t]{2}{*}{ Positive rate } \\
\hline & & & & \\
\hline Gender & & Male:127, Female:123 & & \\
\hline Steatosis & & 98 (39.2\%) & & \\
\hline \multirow[t]{5}{*}{ Fibrosis } & FO & $0(0 \%)$ & & \\
\hline & F1 & $7(2.8 \%)$ & & \\
\hline & $\mathrm{F} 2$ & $102(40.8 \%)$ & & \\
\hline & F3 & $94(37.6 \%)$ & & \\
\hline & F4 & $47(18.8 \%)$ & & \\
\hline $\mathrm{HCC}$ & & $38(15.2 \%)$ & & \\
\hline IL-17F & & 250 & $\begin{array}{l}1246.7 \mathrm{pg} / \mathrm{mL} \\
(0-877.7)\end{array}$ & $30.4 \%$ \\
\hline IL-17A & & 144 & $\begin{array}{l}4.3 \mathrm{pg} / \mathrm{mL} \\
(0-0)\end{array}$ & $6.3 \%$ \\
\hline IL-21 & & 137 & $\begin{array}{l}73.2 \mathrm{pg} / \mathrm{mL} \\
(0-84.7)\end{array}$ & $59.9 \%$ \\
\hline IL-6 & & 153 & $\begin{array}{l}50.6 \mathrm{pg} / \mathrm{mL} \\
(0-0)\end{array}$ & $20.3 \%$ \\
\hline
\end{tabular}

a mean age of 56.6 years; $39.2 \%$ of the patients had steatosis, $100 \%$ had fibrosis, and $15.2 \%$ had HCC (Table 3). The distribution of liver fibrosis stage in this cohort was as follows: F1, 7 cases (2.8\%); F2, 102 cases (40.8\%); F3, 94 cases (37.6\%); and F4 (cirrhosis), 47 cases (18.8\%). As shown in Table 3, IL-17F ELISAs of 250 serum samples obtained from treatment-naïve $\mathrm{HCV}$ patients yielded a mean serum IL-17F concentration of $1246.7 \mathrm{pg} / \mathrm{ml}$. Among the 250 samples, 76 (30.3\%) had IL-17F level > $312.5 \mathrm{ng} /$ $\mathrm{mL}$, the lower detection limit of the ELISA. The expression of inflammatory cytokines, IL-17A, IL-21, and IL-6, which are functionally associated with IL-17F were also measured by ELISA. As some serum samples collected from the HCV patients in 2003-2008 were not sufficient for addition ELISAs, the case numbers $(\mathrm{N})$ for these cytokines were less than 250. IL-17A was detected in very few samples (9/144, $6.3 \%$ ) at a low concentration (mean: $4.3 \mathrm{pg} / \mathrm{mL}$ ). IL-21 was detected in 82/137 samples (59.9\%) at a mean concentration of $73.2 \mathrm{pg} / \mathrm{mL}$, and IL-6 was detected in 31/153 samples $(20.3 \%)$ at a mean concentration of $50.6 \mathrm{pg} / \mathrm{mL}$.

The correlation efficiency between serum IL-17F levels and chronic hepatic diseases, including hepatitis, steatosis, fibrosis, and HCC was evaluated as shown in Table 4.
Serum alanine aminotransferase (ALT) which is usually elevated during liver inflammation was used as an index for hepatitis in Table 4. When the patients were separated into mild (F1 and F2) and severe (F3 and F4) fibrosis groups for statistical analysis, serum IL-17F level was correlated with fibrotic severity (rho $=0.127, p=0.045$ ) (Fig.2). Levels of cytokines IL-17A and IL-21 had strong (rho $=0.547, p<0.0001)$ and moderate (rho $=0.218$, $p=0.011)$ correlations, respectively, with IL-17F in the HCV patients. However, serum IL-17A and IL-21 level were not significantly associated with fibrosis or other $\mathrm{HCV}$-associated diseases by statistical analyses.

\section{Discussion}

The present study showed that a higher percentage of $\mathrm{HCV}$-associated HCC tissues than of NBNC-HCC tissues were found to have IL-17F mRNA expression. IL-17F mRNA expression was higher in tumor tissue than in non-tumor counterparts. Furthermore, the serum IL-17F levels were elevated in $\mathrm{HCV}$-infected patients with severe liver fibrosis than in patients with mild liver fibrosis.

Chronic HBV, HCV infection and steatohepatitis are common causes of liver fibrosis [26-28]. The relevance of Th17 and IL-17 in liver fibrosis has been studied in human patients and mouse models [29,30]. Chang et al. showed that circulating and liver-infiltrating Th17 cell levels correlate with severity of liver inflammation [19]. However, serum IL-17A levels are low in HCV patients and appear not to correlate with $\mathrm{HCV}$-related fibrosis [31]. In our HCV cohort study, the mean serum IL-17F level was much higher than that of IL-17A. The severity of liver fibrosis in $\mathrm{HCV}$ patients was associated only with IL-17F, but not with IL-17A, suggesting that IL-17F might be a better biomarker than IL-17A for HCVassociated fibrosis progression. The serum IL-17F level did not have a significant correlation with serum alanine aminotransferase activity in the HCV cohort, suggesting that IL-17F elevation was not associated with acute liver inflammation.

IL-17A and IL-17F have highly homologous amino acid sequences, bind the same receptor, and activate similar proinflammatory responses, but these two cytokines differ in tissue distribution and receptor binding affinity [32]. In the present $\mathrm{HCV}$ cohort, more serum samples were positive for IL-17F than with IL-17A, and

Table 4 Correlations of serum IL-17F level with the HCV infection-associated liver diseases and other proinflammatory cytokines

\begin{tabular}{|c|c|c|c|c|c|c|c|c|}
\hline & & ALT & Steatosis & Fibrosis $^{c}$ & $\mathrm{HCC}$ & IL-17A & IL-21 & IL-6 \\
\hline \multirow[t]{2}{*}{ IL-17F } & Correlation Coefficient (r) & -0.094 & 0.012 & $0.127^{a}$ & 0.014 & $0.547^{b}$ & $0.218^{a}$ & 0.009 \\
\hline & Sig. & 0.142 & 0.850 & 0.045 & 0.826 & 0.000 & 0.011 & 0.912 \\
\hline
\end{tabular}

\footnotetext{
${ }^{a}$ Correlation is significant at the 0.05 level (2-tailed)

${ }^{b}$ Correlation is significant at the 0.01 level (2-tailed)

c Fibrosis: stage $>2$

Spearman's rank correlation
} 


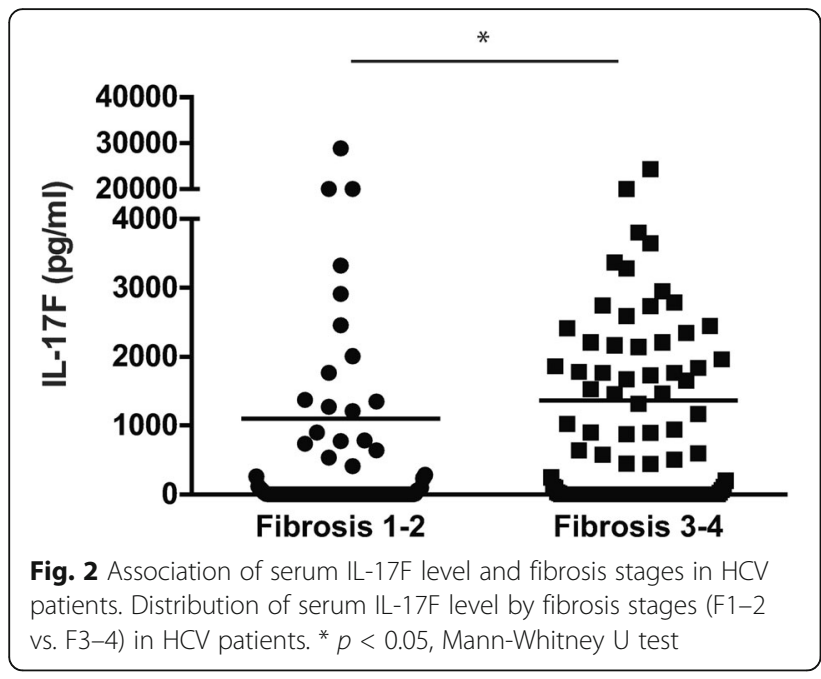

the mean IL-17F level was much higher than the mean IL-17A level. High levels of IL-17F can be due to a high transcription activity, a long protein half-life, or production by different cell types. Given that the expression of serum IL-17A and IL-21, two key cytokines produced by Th17 cells, was associated with IL-17F expression, it is likely that Th17 cells contribute to IL-17F expression. However, in our IL-17F IHC analysis of HCC tissue sections, IL-17F expression was found mainly in hepatocytes. Serum IL-17F in HCV-infected patients may be produced partially by hepatocytes. The stimuli capable of triggering IL-17F expression in hepatocytes and other cell types and the responses activated by IL-17F-dependent signaling in the liver will need to be addressed further.

A pathological role of IL-17 and Th17 in HCC development has been proposed based on recent studies [29, 33-35]. Proinflammatory, anti-apoptosis, and proangiogenesis signals may contribute to the tumor promotion role of Th17 cells and IL-17 in HCC development [29]. The role of IL-17F in HCC remains undetermined. In the present study, we found that IL-17F was elevated in patients with $\mathrm{HCV}$ and advanced fibrosis and that IL-17F mRNA was also elevated in $\mathrm{HCV}$-associated tumor tissue. Notably, the IL-17F protein was found mainly in hepatocytes. The exact function of IL-17F in HCC development needs to be clarified with long-term follow-up cohort study.

\section{Conclusions}

The importance of IL-17F in chronic hepatic diseases was poorly investigated; however, we had found Th-17 associated inflammatory cytokine, IL-17F, which took a major part in severe liver fibrosis and HCC symptoms in HCV patients than other inflammatory cytokines, IL-6, and IL17A. It appears that IL-17F can be a precise biomarker for the diagnosis of liver fibrosis and HCC progression in the future.

\section{Acknowledgements}

We thank Tainan Municipal Hospital and Taiwan Liver Cancer Network for providing patient serum, RNA and tissue sections.

\section{Funding}

This work was supported by National Health Research Institutes (Taiwan), grant number IV-104-pp.-21.

\section{Availability of data and materials}

The datasets generated during and/or analyzed during the current study are available from the corresponding author on reasonable request.

\section{Authors' contributions}

MSW and CHW contributed equally to this work. MSW, CHW, and GYY were in involved in experimental design, data analysis, and manuscript preparation. FCT performed statistical analyses. HJY, YCL, YPK, DJT, and WTT performed related experiments. All authors read and approved the final manuscript.

\section{Ethics approval and consent to participate}

The studies were approved by Institutional Review Board committees of Tainan Municipal Hospital (IRB1011205) and National Health Research Institutes (EC1000801)

\section{Consent for publication}

Not applicable.

\section{Competing interests}

The authors declare that they have no competing interests with the contents of this article.

\section{Author details}

${ }^{1}$ National Institute of Infectious Diseases and Vaccinology, National Health Research Institutes, 35 Keyan Road, Zhunan, Miaoli County 35053, Taiwan. 2Division of Gastroenterology, Tainan Municipal Hospital, Tainan 70173, Taiwan. ${ }^{3}$ Center of Infectious Disease and Signaling Research, National Cheng-Kung University, Tainan 70101, Taiwan.

Received: 3 May 2017 Accepted: 19 July 2017

Published online: 26 July 2017

\section{References}

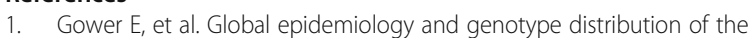
hepatitis C virus infection. J Hepatol. 2014:61 (1 Suppl):S45-57.

2. Friedman SL. Liver fibrosis - from bench to bedside. J Hepatol. 2003; 38(Suppl 1):S38-53.

3. Fattovich $\mathrm{G}$, et al. Hepatocellular carcinoma in cirrhosis: incidence and risk factors. Gastroenterology. 2004:127(5 Suppl 1):S35-50.

4. Donato F, et al. Alcohol and hepatocellular carcinoma: the effect of lifetime intake and hepatitis virus infections in men and women. Am J Epidemiol. 2002;155(4):323-31.

5. El-Serag HB. Hepatocellular carcinoma and hepatitis $C$ in the United States. Hepatology. 2002;36(5 Suppl 1):S74-83.

6. Tesmer LA, et al. Th17 cells in human disease. Immunol Rev. 2008;223:87-113.

7. Hohl TM, Rivera A, Pamer EG. Immunity to fungi. Curr Opin Immunol. 2006; 18(4):465-72.

8. Komiyama $Y$, et al. IL-17 plays an important role in the development of experimental autoimmune encephalomyelitis. J Immunol. 2006:177(1):566-73.

9. Miossec P. IL-17 and Th17 cells in human inflammatory diseases. Microbes Infect. 2009:11(5):625-30.

10. Dardalhon $\mathrm{V}$, et al. Role of Th1 and Th17 cells in organ-specific autoimmunity. J Autoimmun. 2008:31(3):252-6.

11. Wei $L$, et al. $I L-21$ is produced by Th17 cells and drives $\mathrm{LL}-17$ production in a STAT3-dependent manner. J Biol Chem. 2007;282(48):34605-10.

12. Bettelli $\mathrm{E}$, et al. Reciprocal developmental pathways for the generation of pathogenic effector TH17 and regulatory T cells. Nature. 2006; 441(7090):235-8.

13. Veldhoen $\mathrm{M}$, et al. TGFbeta in the context of an inflammatory cytokine milieu supports de novo differentiation of IL-17-producing T cells. Immunity. 2006;24(2):179-89. 
14. Iwakura $Y$, et al. Functional specialization of interleukin-17 family members Immunity. 2011;34(2):149-62.

15. Lemmers $A$, et al. The interleukin-17 pathway is involved in human alcoholic liver disease. Hepatology. 2009;49(2):646-57.

16. Lan RY, et al. Hepatic IL-17 responses in human and murine primary biliary cirrhosis. J Autoimmun. 2009;32(1):43-51.

17. Yasumi $Y$, et al. Interleukin-17 as a new marker of severity of acute hepatic injury. Hepatol Res. 2007;37(4):248-54.

18. Tan Z, et al. IL-17A plays a critical role in the pathogenesis of liver fibrosis through hepatic stellate cell activation. J Immunol. 2013;191(4):1835-44.

19. Chang Q, et al. Th17 cells are increased with severity of liver inflammation in patients with chronic hepatitis C. J Gastroenterol Hepatol. 2012;27(2):273-8.

20. Takatori $\mathrm{H}$, et al. Lymphoid tissue inducer-like cells are an innate source of IL-17 and IL-22. J Exp Med. 2009;206(1):35-41.

21. Ishigame $\mathrm{H}$, et al. Differential roles of interleukin-17A and $-17 \mathrm{~F}$ in host defense against mucoepithelial bacterial infection and allergic responses. Immunity. 2009;30(1):108-19.

22. Chang IC, et al. The hepatitis viral status in patients with hepatocellular carcinoma: a study of 3843 patients from Taiwan liver cancer network. Medicine (Baltimore). 2016;95(15):e3284.

23. Kim S, Kim T. Selection of optimal internal controls for gene expression profiling of liver disease. BioTechniques. 2003:35(3):456-8. 460

24. Livak KJ, Schmittgen TD. Analysis of relative gene expression data using real-time quantitative PCR and the 2(-Delta Delta $C(T))$ method. Methods. 2001;25(4):402-8

25. Grivennikov $\mathrm{S}$, et al. IL-6 and Stat3 are required for survival of intestinal epithelial cells and development of colitis-associated cancer. Cancer Cell. 2009;15(2):103-13.

26. Yilmaz B, et al. Chronic hepatitis B associated with hepatic steatosis, insulin resistance, necroinflammation and fibrosis. Afr Health Sci. 2015;15(3):714-8.

27. Sebastiani G, Gkouvatsos K, Pantopoulos K. Chronic hepatitis $C$ and liver fibrosis. World J Gastroenterol. 2014;20(32):11033-53.

28. Bataller R, Brenner DA. Liver fibrosis. J Clin Invest. 2005:115(2):209-18.

29. Hammerich L, Heymann F, Tacke F. Role of IL-17 and Th17 cells in liver diseases. Clin Dev Immunol. 2011;2011:345803.

30. Meng F, et al. Interleukin-17 signaling in inflammatory, Kupffer cells, and hepatic stellate cells exacerbates liver fibrosis in mice. Gastroenterology. 2012;143(3):765-76. e1-3

31. Foster RG, et al. Interleukin (IL)-17/IL-22-producing T cells enriched within the liver of patients with chronic hepatitis C viral (HCV) infection. Dig Dis Sci. 2012;57(2):381-9.

32. Yang $\mathrm{XO}$, et al. Regulation of inflammatory responses by IL-17F. J Exp Med. 2008;205(5):1063-75.

33. Zhang JP, et al. Increased intratumoral IL-17-producing cells correlate with poor survival in hepatocellular carcinoma patients. J Hepatol. 2009;50(5):980-9.

34. Li J, et al. Interleukin 17A promotes hepatocellular carcinoma metastasis via NF-kB induced matrix metalloproteinases 2 and 9 expression. PLoS One. 2011;6(7):e21816.

35. Kuang DM, et al. Activated monocytes in peritumoral stroma of hepatocellular carcinoma promote expansion of memory T helper 17 cells. Hepatology. 2010:51(1):154-64.

\section{Submit your next manuscript to BioMed Central and we will help you at every step:}

- We accept pre-submission inquiries

- Our selector tool helps you to find the most relevant journal

- We provide round the clock customer support

- Convenient online submission

- Thorough peer review

- Inclusion in PubMed and all major indexing services

- Maximum visibility for your research

Submit your manuscript at www.biomedcentral.com/submit

C Biomed Central 